\title{
The Role and Value of Geospatial Information and Technology in a Pandemic
}

\author{
Abbas Rajabifard, Daniel Paez and Greg Foliente
}

\section{$1.1 \quad$ Introduction}

The coronavirus COVID-19 pandemic is the defining global crisis of our time and the most devastating challenge the world has faced since World War II, having a profoundly deep impact on the way we perceive our world and our everyday lives. While beginning as a health crisis, it has grown fast in just over few months to be an unprecedented socio-economic and environmental crisis that has spread to every continent and country. As a result, the world has been facing unprecedented social and economic changes and challenges, across all industries and sectors, at every scale (from local, to national and to global level). The COVID-19 virus is a global pandemic, as all our societies are connected, in which no country or jurisdiction is left unscathed as a result of this situation.

This book aims at covering these disciplinary intersections that happen when Geographic Information Systems (GIS) and location intelligence are used in action to respond to the crises and plans for recovery. The experiences and the information included in this book will be a learning tool for communities to being prepared, making the right decisions, and keeping informed to be able to improve community resilience and respond to future crisis.

During a pandemic political borders mean nothing, and every society has been impacted in some way. In June 2020, the World Bank forecast that global gross domestic product (GDP) would shrink by $5.2 \%$ [1] in 2020, with recessions in both advanced and emerging market economies. The devastating effects on the global economy will take years to overcome, as governments around the world grapple with the situation and make impossible decisions that aim to meet both the health and economic wars faced as a result of the virus in an attempt to save lives but also limit the financial distress faced by the global economy.

We are now living with a new norm of practice, and as each day passes, people all over the world are not only losing their lives and their health, but also employment, their livelihoods, connections with their friends and family, with no real sense of when life will return to a familiar normality. In this context, good data and statistics, in particular location information and related attributes are key for our resilience and data infrastructure for sustainable future. These data are crucial as they make essential contributions to our strategic pathways on our wider public safety. In a global crisis, our leaders and decision-makers require the fast delivery of information that is accessible and reliable, so urgent and effective responds to COVID-19 are available to make the right choices, manage the complexity and uncertainty and thus lead the world to a more resilient and sustainable future.

From a location information (location intelligence) perspective, the COVID-19 pandemic has highlighted to the world the unequivocal importance and need for geospatial information, enabling technologies, and clear and concise information for governments that enable decision-makers to keep their citizens safe, plan for the future and protect the most vulnerable in our communities.

So much of managing a pandemic is inherently a geographic and location issue. In order to manage outbreaks, perform contact-tracing and enable a robust community response to stop the spread, spatial information is required, and location information is paramount. The social and environmental factors, including population density and age, employment and lifestyle, all influence patterns of disease occurrence and prevalence.

As such, location information, mapping and related analytical tools such as GIS tools are widely used by health departments, safety and emergency management authorities and wider professionals around the 
world for gathering and analysing data to support informed decisions. The use of location intelligence and GIS for understanding this outbreak and its relationship to infrastructure, population, businesses and other location-based information requires both a clear understanding of the relevant geospatial principles and the relevant aspects of data monitoring, planning and mapping.

\subsection{Critical Role of Location Information}

The COVID-19 pandemic is proving a need for a collaborative geospatial infrastructure that will help us better understand the crisis at hand at both a local and global scale, as well as better prepare us for future pandemics worldwide. There is a growing need for real-time maps, and location information to track and share location data, and as such, a geospatial-enabled platform, along with data analytics tools and solutions play a critical role in assisting the pandemic's front lines and keeping the general public informed and prepared.

GIS for example has always been a tool in managing the response to large-scale disasters by using location technology that helps to understand the situation at hand, develop a response and prepare a road to recovery. A spatial team is of paramount importance to help create the maps, data, apps, analysis and dashboards that are required for emergency management and resource allocation.

Early in 2020, there was an extraordinary effort around the globe where GIS practitioners in every country began to work together, sharing information that helped us all grasp the situation at hand and also enabled the decision-makers in their response to the virus at local, national and global levels. The Johns Hopkins Coronavirus Dashboard, based on Esri software, was originally built by a handful of people but has evidently grown into the primary tool used to track and monitor the virus, being viewed "nearly a trillion times only a few months into the pandemic" as stated by Esri CEO, Jack Dangermond.

Location information plays a critical role managing the spread of the virus and protecting our communities by providing the tools required in collecting vital data, mapping the current crisis, simulating the results from modelling response variables, contact tracing and determining hot spots, managing high-risk locations and distributing help where it is needed most. Spatial experts around the world have come together and shared critical information that has transpired into an unprecedented effort to manage the COVID-19 pandemic in a way that has not been evident in our history. This effort has been on a global scale and as such we have evolved into a world that supports a connected and global GIS. In our new interconnected world, we all benefit from this phenomenal transformation that impacts every government, industry and citizen, where we can make better and more informed decisions about our future.

\subsection{Impact of COVID-19 on the Sustainable Development Goals (SDGs)}

The Sustainable Development Goals (SDGs), as outlined by the United Nations as part the global plan to create a better future for all, now play an even more critical role in providing a framework tool that puts health and prosperity at the core of the road to recovery for all nations and all people around the globe. The COVID-19 pandemic has emphasised the challenges facing governments from taking the extraordinary steps needed to attain the SDGs. The vulnerabilities and obstacles that exist in different countries when working towards achieving the SDGs are even more prominent now, including socio-economic gaps, structural inequalities, systemic challenges and risks, as well as a lack of timely fundamental data and enabling technologies to measure and monitor what is happening where, when and how (UN-GGIM White paper, COVID-19). The United Nations Committee of Experts on Global Geospatial Information Management (UN-GGIM) has been taking steps to explore how geospatial information has been used to support national response efforts, and the influence and impact of the geospatial data ecosystem in responding to the global COVID-19 pandemic.

This unprecedented time has also been the first time where geospatial data location intelligence from all around the world has been collected, visualised and made available from local to global levels, through numerous dashboards, including from the WHO and Johns Hopkins University to name a few. These geospatial visualizations clearly communicate the situation and guide decision-making, all through location-based data, and demonstrate that when the geographic dimension is considered, information can be communicated in a clearer, more effective manner, not only to governments but to every individual citizen on the globe.

Of significant importance, contact tracing applications have demonstrated hope by identifying outbreak hotspots, providing those in power with a means to identify, inform and subsequently treat those infected, limiting the spread of the pandemic among the population. In order to achieve successful contact tracing, 
there needs to be high-level access to geospatial information, which can be queried and classified by age, gender, demography and geography, at every level.

There is an urgent need for the work and issues raised by the UN-GGIM to be addressed and made available across the globe, including data availability, core reference data, interoperability, common geographies, integration of statistics and geography, privacy and confidentiality, the relevance of the Integrated Geospatial Information Framework (IGIF).

With the Integrated Geospatial Information Framework (IGIF) at its core, the UN-GGIM has published findings on what components are required to respond to the COVID-19 crisis, such as leadership, governance, legal and policy, data and technology. The work demonstrates that a clear connection to the IGIF will enable countries to respond in a more timely manner in a crisis where time is critical. Through its initiatives, the UN-GGIM aims to bring the international geospatial community together, along with the the statistical, public health, and development sectors to fight the current pandemic and to build a more resilient and robust global community.

As the IGIF provides a holistic and inclusive Framework, applicable to all countries and in all situations, it is able to be applied to the global COVID-19 pandemic. As an example, the IGIF is highly beneficial in disaster response, where data sharing and geospatial information are critical. Similarly, the response to COVID-19 also requires the ability to share integrated geospatial information in real-time. This ensures that the same information will be delivered to all the key players of the response at the same time. This is also critical in the responding to the aftermath of any disaster, where geospatial determines what happens next and where actions are needed. Outlined in the UN-GGIM white paper publications, the IGIF focuses on geospatial information that is integrated with any other meaningful data to solve societal and environmental problems, acts as a catalyst for economic growth and opportunity, and to provide understanding and benefit from a countries development priorities and the SDGs. While the IGIF has anchored the geospatial response to COVID-19, it is important to note that it was not developed as a response to COVID-19, only as an enabling mechanism to achieve the ambitious targets set by the SDGs. However, its usefulness has provided countries with a basis for leveraging geospatial information, either to integrate existing capacity, or through developing new capability. As a result, the IGIF has demonstrably enabled countries to determine their national geospatial response to COVID-19. The IGIF has nine strategic pathways, influenced by Governance, Technology and People, that work together as a basis and guide for developing, integrating, strengthening and maximizing geospatial information management and related resources in all countries, resulting in tangibly enabled countries to leverage geospatial information in their COVID-19 response.

The most vulnerable countries around the world that have faced the greatest challenges with achieving the SDGs, now face the greatest challenges with the COVID-19 pandemic. Collecting high-quality geospatial information as well as producing, analysing and using timely and reliable location-based data is often not possible, and in many countries where may exist in some form somewhere, it is often not discoverable, structured, interoperable or standardized. It cannot be readily accessed, shared, and more importantly, integrated with other data for decision-making.

Both in attaining SDGs and responding to the pandemic, geospatial information is the key integrator that enables informed decision-making, as well as enabling visualisation and analysis required to communicate key data to decision-makers and the general public. As such, the past few months have been a testament to the importance of collectively developing mechanisms, mobilizing resources and strengthening the global capacity of geospatial information. In this way we can then meet the challenges of COVID-19, and work to build back a stronger path forward on the road to meet the needs of the SDGs in the future.

The UN-GGIM has provided a platform for the global geospatial community and demonstrated its importance to respond to the challenge of COVID- 19. It has become evident how urgent and critical it is to have a multifaceted system that mobilises resources and ensures that all agencies, including government and the private sectors, have access to geospatial information in order to best manage the pandemic, and work towards the SDGs. Implementing the IGIF has been an important step in developing dashboards and communicating the spread and developments of COVID-19.

There is no denying that the pandemic has been a setback in achieving the SDGs around the globe, however it is also a time of great opportunity and growth as there has never been a time in history where people from every corner of the world are working together to bring solutions and advancements to better our health, our communities, our environment and the economy. This particularly effects developing countries, which may for the first time have access to geospatial data and tools that will not only benefit the management of the pandemic in such countries, but also provide their agencies with digital data for challenges that lie ahead.

\subsection{Digital Innovation During a Pandemic}

In times of great pressure that require solutions to distinct challenges, scientists and engineers are required to fast develop methods, technologies and innovation, and create new good practices. The pandemic has illustrated that novel innovations including dashboards and entirely new processes can be fast tracked and implemented, in a matter of weeks, rather than years. As a result, governments are able to make better and 
more informed decisions by understanding where the risks exist. Emerging technologies such as Artificial Intelligence (AI) help to expedite the development of a vaccine, and predict the most effective public health measures through simulation and help move much of our lives to an online environment, where we can maintain our economic and education systems to continue employment and schooling as well as our connection to one another.

This also highlights the gap across various social groups in digitally isolated countries and regions. The COVID-19 crisis reminds us that we should nurture the socially beneficial applications of digital technologies and work to improve accessibility and usage in the countries and areas which require the greatest leverage and aid. There are many examples of digital innovation across the globe including in the United Nations Educational, Scientific and Cultural Organization (UNESCO) and the International Business Machines Corporation (IBM) that support the development and deployment of public health mobile applications to help manage the pandemic by providing informed data-driven decisions and social distancing measures. There is also a push to enhance access to the Internet across the globe through UNESCO that aims to foster collaboration and partnership in developing countries.

Governments around the world are also now spending record funding (US\$10.5t [2] and rising) to manage the COVID-19 pandemic which means there is a real opportunity for partnerships with businesses to emerge and fast track innovations that lead an advancement in technologies, and how we manage public health as well as how we restore the environment.

However, governments, businesses and the financial services sector need to ensure that investments in innovation create opportunities, reduce inequality and lead to net gains in employment. And governments can do more than just fund innovation; they can ensure learning is plugged back into the state. Digitalization lessons from industry, for example, can be applied to public sector services to deliver better and more effective access, while preserving provision in the face of budget deficits.

\subsection{Collaboration and Engagement}

Throughout the pandemic, we've seen the development of extraordinary models of collaboration that have resulted in the largest exchange of scientific data in history. It has been remarkable to see competitors work together in joint initiatives. And although there have been some level of disagreement amongst governments and international agencies, we have also observed efficient and effective collaborations between the state and private sectors to deliver technological and medical solutions at speed. This time during the pandemic has been an example of just how much we can achieve as international citizens, when we all focus on a common goal.

There has, however, been indications of an increase in the social divides, similar to the aftermath of the global financial crisis (GFC), where the recovery of many markets were uneven and the people who struggled the most fell further into crisis. The impact on jobs around the world has been dire: the International Labor Organization (ILO) estimates a decline in global working hours in the second quarter of 2020 of around $10.7 \%$ (equivalent to 305 million full-time jobs) relative to the last quarter of 2019 [3]. And the workers in poorly paid jobs, casual contracts or who are self-employed are increasing in numbers making them more vulnerable to layoffs during the economic downturn and with less access to social security safety nets. An inequality in healthcare, including access to medical attention, testing availability, access to intensive care and medication, has also been one of the crucial issues of the pandemic observed around the world. Further to this, the recent occurrence of protests that has spread from the United States across Europe to Australia demonstrates the urgent need for inequality to be addressed.

There is also the likelihood that another pandemic may happen in the future, and as such, building a close coordination between central government and local governments, and ensuring closer international collaboration, together with the sharing of digital innovation and technological advancements are of increasing importance for a sustainable future.

\subsection{Opportunities Emerging from the Pandemic}

Our wider society through governments, the private and the public sectors have a unique and strategic opportunity to shape the recovery and the future economy in such a way that will ensure a more sustainable and more equitable outlook for all. There is now a chance to reframe the future by building a fairer, more prosperous society founded on greener, more resilient, productive economies. While every country and region will have different priorities and starting points, the starting principle for all governments should seek to build more resilient economies while also tackling the climate change challenges and social inequalities.

Social equity is a priority that underpins sustainable economic prosperity. The opportunity to address social inequality, improve outcomes for all citizens, including fairer access to employment is an opportunity 
during this time that should not be missed. A sustainable recovery is not just about creating jobs; it's about creating high-quality, accessible jobs that improve peoples' lives, which in turn creates more stable societies. The pandemic has also forced the adoption of new ways of working. Organizations must reframe their work and the role of offices in creating safe, productive and enjoyable jobs and lives for employees.

The environmental opportunities in the pandemic that promote a green recovery is clear. The vast majority of the world's total GDP is moderately or highly dependent on a stable environment and is therefore susceptible to disruption due to climate change [4]. Without action to address climate change, an additional 100 million people could be pushed into poverty by 2030 and 143 million in just three regions could be displaced [5]. In the wake of COVID-19, governments and companies have an opportunity to plot greener, fairer and more sustainable days ahead. The pandemic has also raised environmental fears to the public too, as the enforced pause on industrial pollution and travel has resulted in cleaner air where $\mathrm{CO}_{2}$ emissions are now predicted to be at the lowest level in 14 years [6]. United Nations studies also now estimate that a move to low-carbon, resilient economies could possibly create 65 million new jobs between now and $2030[7]$.

\subsection{Moving Forward from the Pandemic}

With the lessons that we are all learning from this pandemic, our leaders in the public and private sectors, in academia must look to rise from the challenges we face and identify opportunities, in which we can find new ways to keep growing and building resilient and sustainable communities, and infrastructure.

Through the devastation, the COVID-19 pandemic has also demonstrated the interconnected nature of our world and communities, and further highlights the United Nations notion, that no one is safe until everyone is safe. We must act in solidarity with our communities all around the world, in order to save lives and overcome the devastating impacts that this virus has, not just to our health, but socially and from an economic standpoint. By demonstrating strong partnerships, we can grow our sense of humanity and inspire all generations to hope for a healthier and more prosperous future for all, where we learn from navigating through this challenging journey and work together to build greater resilience in every community.

\subsection{This Book, Objectives, Chapter Outline}

This book brings together the expertise of leading professionals, practitioners and academics in different related fields for managing pandemic, in particular experts in the field of geospatial industry, and policy-makers and their perspectives to share their experiences and approaches to respond to the COVID-19 pandemic, and they share their lessons for any similar pandemic in future in order to improve community resilience. The themes and objectives of the book are in line with the critical challenges that our global community is facing. It is also in line with challenges, gaps, and the work plan of the UN-GGIM Academic Network.

Over 120 authors from regions all around the world contributed to the production of this book, including contributions from more than 30 countries and country case studies. 


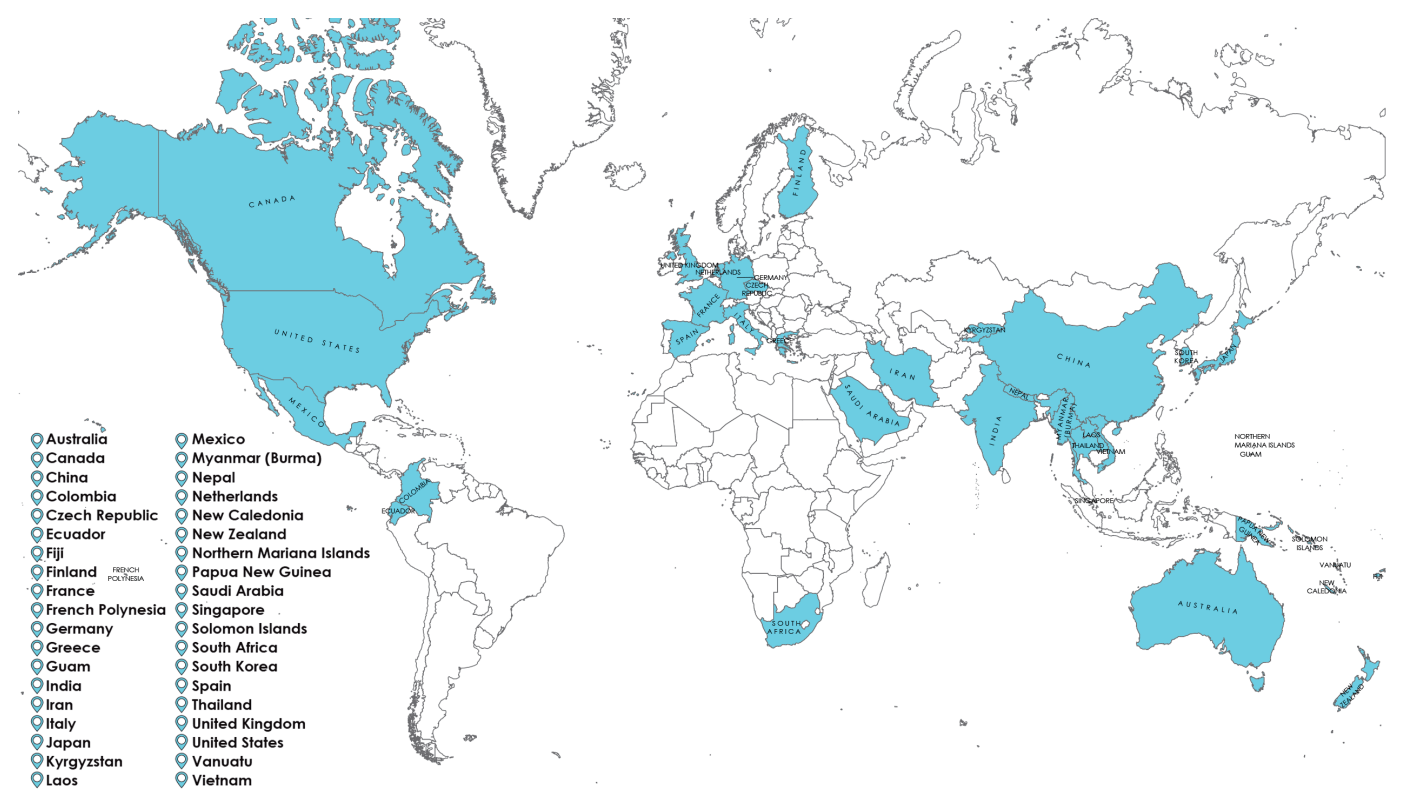

\section{FIGURE 1.1}

Contributions and country case studies

The authors have shared their observations, research and best practices in their jurisdictions and organizations that are relevant to professional lines of work or supporting training and teaching modules focusing on COVID-19 applications. This will provide the foundation that would help to reassess the field, affirm successful approaches and point to future possibilities.

The book brought together two types of contributions, scientific chapters, and industry and practitioner observations, structured into 47 chapters in 5 parts. The authors have worked together in hope of taking steps towards achieving more sustainable, safe and resilient future. In doing so, the book will address the following objectives:

- Review foundational aspects of geospatial sciences and technologies for supporting intelligent decision-making for pandemic management.

- Identify a coherent set of tools, guidelines or standards to help researchers, data producers and practitioners and authorities utilize geospatial information for decision-making during various pandemic phases.

- Provide a resource on current best practices for utilizing location intelligence for local, regional, national and global level pandemic management.

- Reflect on the lessons learnt from COVID-19 pandemic, and present a forward-looking collection of ongoing research, development and practice, with an emphasis on the role of location and geospatial science, that can improve the resilience of community, society, economy and environment.

- Provide a medium for presenting the challenges, solutions, opinions and insights from different stakeholders regarding their experience through the pandemic.

The beginning chapters of this book provide an overview of the principles and foundations for geospatial science and technologies, followed by an outline of current approaches for pandemic management and reflections for ongoing development and future prospects for geospatial data in building a more resilient community, society, economy and environment. This book is unique in that it contains observations from various countries and industries, including the challenges they faced, the solutions they came across, the opinions and insights they had during this crisis. It will help build tight bonding between industries and academics and drive more industry-oriented research opportunities.

In Chapter 2, Keith Clifford Bell and Vladimir V. Evtimov draw from the experience of the World Bank and the Food and Agriculture Organization (FAO) to discuss a way forward to ensure the resilience of countries, cities and communities through authoritative geospatial information and land administration.

Maria Antonia Brovelli and Serena Coetzee explore open geospatial data for responding to the COVID-19 challenge in Chapter 3, by examining what data is useful when studying the spatio-temporal spread of the virus and investigating the availability of such open data. In Chapter 4, Mohammad Reza Mobasheri reports on the role of remote sensing in the detection, evaluation and mapping factors relating to public health. The chapter focuses on an introduction to the importance and use of remote sensing through an examination of successful remote sensing applications. 
David Green et al. explore the potential of drone technology during pandemics in Chapter 5. The study includes an overview of current drone technology and future developments, as well as investigation into the issues facing successful mainstream implementation of the use of drones for such applications, and the problems that need to be overcome to allow this technology to become mainstream. In Chapter 6 , Piret Veeroja and Greg Foliente look at the role of social and built environments on social interactions in Melbourne prior to, and during, the COVID-19 pandemic and discuss possible social and built environment interventions to increase social interactions.

Farhad Laylavi reports on a study in Chapter 7, which aims to contextualise social vulnerability to pandemic situations. The study presents the preliminary indicators of social vulnerability and presents a discussion of the implications, limitations and future work. In Chapter 8, Renate Thiede and Inger Fabris-Rotelli present an algorithm for detecting informally developed roads in satellite images and quantify the uncertainty associated with the results. Such roads play an important role in the development of COVID-19 response strategies in developing countries.

In Chapter 9, Rafael Ponce Urbina et al. showcase a holistic view of the uses of GIS to monitor, analyse and disseminate knowledge pertaining to maritime geospatial data during the COVID-19 pandemic. Mark Stevenson et al. outline recent research in city design and the transmission of COVID-19 in Chapter 10. The chapter highlights the role of city design in virus transmission, particularly in high-density road networks and public transit.

In Chapter 11, Felicia N. Huang et al. discuss social media use and community resilience development during the COVID-19 pandemic in Singapore. Lesley Arnold, Zaffar Sadiq Mohamed-Ghouse and Tony Wheeler report on the role of professional bodies during a pandemic in Chapter 12, with a focus on the role of the Surveying and Spatial Sciences Institute (SSSI) in Australia. In Chapter 13, Peter Mooney et al. explore OpenStreetMap (OSM) data use cases during the early months of the pandemic. The chapter contributes to the knowledge on how volunteered geographic information (VGI) initiatives such as OSM respond and are used or accessed during a global crisis such as COVID-19.

In Chapter 14, Pravin Kokane et al. explore the utilisation of geospatial network analysis techniques for optimal route planning during the pandemic. Chryssy Potsiou discusses the critical issue of formalising informal settlements to empower residents against COVID-19 and other disasters in Chapter 15. The chapter reports on how geospatial experts can provide the means to support governments to identify and empower those most vulnerable to the pandemic, and the measures that have taken place. Abbas Rajabifard et al. provide a discussion around spatially enabled platform supporting managing pandemic, and also review of the applications and systems in place for such a spatially enabled COVID-19 in Chapter 16.

In Chapter 17, Neda Kaffash Charandabi and Amir Gholami propose a global model to help determine the important periods of each country, predict confirmed cases and to discover spatio-temporal hot/cold spots based on wavelet and neural networks. In Chapter 18, Michael Batty et al. give a report on London in Lockdown, discussing the issues of mobility in the pandemic city.

Rosario Casanova et al. in Chapter 19 discuss the Americas geospatial response to COVID-19 through an overview and analysis of the implementation of UN-GGIM global frameworks, as well as a discussion of the gaps and challenges faced. In Chapter 20, Marije Louwsma and Hartmut Müller explores spatio-temporal information management to control the pandemic in Europe. Zhixuan (Jenny) Yang reports on online higher education in Chapter 21, with a focus on how online education facilitated by Information and Communication Technology (ICT) in China has been implemented during the COVID-19 pandemic. The chapter analyses the experience of online education, and uses a case study to evaluate the critical elements involved.

In Chapter 22, Nadia Abbaszadeh Tehrani et al. provide a time-series analysis of COVID-19 in Iran, from a remote sensing (RS) perspective. The chapter discusses the novel approach that utilises remote sensing data to monitor the pandemic at a national level, as well as examining the applicability of RS coupled with time-series analysis for the study area. Alize le Roux et al. present the COVID-19 Vulnerability Dashboard for South Africa in Chapter 23, which maps out the vulnerability to the pandemic across the whole of South Africa. The study aims at helping local authorities and other stakeholders with disaster risk reduction and evidence-based decision-making.

Bola Michelle Ju et al. describe the rapid development of location-based apps in Chapter 24, focusing on the seven applications which saved lives during the pandemic in South Korea. In Chapter 25, Sultana Nasrin Baby et al. report on a spatial analysis of urban parkland and COVID-19 in the City of Whittlesea, in Victoria, Australia. Phil Bright and David Abbott describe the impact of COVID-19 to the Pacific Island Countries and Territories (PICTs) in Chapter 26.

In Chapter 27, Freya M. Shearer et al. present evidence to inform evolving COVID-19 response planning by analysing how Australians were thinking, feeling and behaving in response to the so-called "first wave" of the COVID-19 epidemic and the associated public health measures. Raul Marino et al. present the results of an investigation relating to the lack of access to public space and social interactions in three Latin-American cities in Chapter 28. David J. Coleman and Prashant Shukle provide a case study in Chapter 29 on how geospatial information and technologies were used in Canada. They highlight observations made in the early stages of Canada's response to the pandemic and provide a critical discussion and opportunities for future development and cooperation.

Milan Konecny et al. discuss the roles of geospatial intelligence in addressing COVID-19 challenges in Czechia in Chapter 30. They present three approaches to improving response to COVID-19, including visual analytics; Tracking and analysis solutions; and decision support systems. Carmen Martin and François Pérès provide an observation and analysis of COVID-19 in France in Chapter 31, with a focus on the multi-phase and multidimensional approach to a complex societal imbalance. 
In Chapter 32, Richard Simpson explores the importance of a worldwide infrastructure when managing complex pandemic scenarios. He investigates the challenges faced with uncoordinated international cohesion and the accumulative crisis humanity faces as a result. In Chapter 33, Jamie Leach as an observation explores the Open Data Pandemic, and discusses the issues that arise in data sharing around the globe.

In Chapter 34, Menno-Jan Kraak addresses the challenges of mapping COVID-19, and addresses two issues including the professional cartographic design challenges and the most common cartographic mistakes witnessed in the media. In Chapter 35, Alice Kesminas also provides an observation by discussing the importance of engagement to build smarter and more resilient communities. She addresses the role that geospatial information and technology play in building community resilience and discusses the challenges faced to successfully plan and manage resilient communities.

In Chapter 36, Frank Friesecke investigates how the coronavirus could change urban planning. The study explores the changes in how we operate schools, retail, work environments and presents a first vision of the direction in which the city of the future could develop towards in light of COVID-19 and other potential pandemics in the future. In Chapter 37, Hossein Mokhtarzadeh shared his view and investigates the move towards agile strategies during the pandemic. This chapter reports on a observational study completed through a number of interviews with experts discussing how they cope with a global crisis.

Kirsikka Riekkinen provides observations from Finland in Chapter 38, with a focus on the opportunities for digitalisation, and the use of geographic information in the Finnish context. Efthimios Bakogiannis et al. report on the possible future of Greek cities in Chapter 39, with a focus on what the cities will look like after the pandemic period with a case study review analysis and best practice assessment.

Saied Pirasteh et al. explore the COVID-19 pandemic challenges and impacts on the Sustainable Development Goals 2030 in Chapter 40. The chapter discusses an understanding of the pandemic and its influences on SDGs 2030, from an Indian perspective. Malcolm Campbell et al. discuss the value of a policy-response research model in Chapter 41. The authors discuss the GeoHealth Laboratory (GHL) research model that is based on a relationship contract funding model in New Zealand.

Mark Allan outlines the lessons learned from management of the Melbourne COVID-19 pandemic in Chapter 42. His contribution chapter presents some observations made and discusses a future of co-existence with the virus.

In Chapter 43, Hassan M. Khormi explores spatial modelling concepts in Saudi Arabia as an observation. The study reports on how the Saudi authorities implement GIS concepts in controlling the spatial risks of the pandemic. Carmen Femenia-Ribera and Gaspar Mora-Navarro examine COVID-19 in Spain with a focus on the use of geospatial information in Chapter 44. Nathaniel Carpenter and Anna Dabrowski discuss the lessons learned from the COVIDSafe app in Chapter 45, a track and trace technology designed for the Australian context. The chapter focuses on understanding conditions for successful implementation of track and trace technologies. Arturo Ardila-Gomez explores sustainable transport as a key pillar to community resilience during the COVID-19 pandemic in Chapter 46.

The book then concludes in Chapter 47, with synthesis of key lessons and future work to improve our planning and preparation for the next pandemic, focusing on geospatial information and related techno-social innovations for enhanced community resilience.

\section{References}

[1] https://www.worldbank.org/en/publication/global-economic-prospects

[2] https://blogs.imf.org/2020/05/20/tracking-the-9-trillion-global-fiscal-support-to-fight-covid-19/

[3] ILO Monitor: COVID-19 and the world of work. Fourth edition. https://www.ilo.org/global/about-the-ilo/ newsroom/news/WCMS_743036/lang--en/index.htm

[4] World Economic Forum. (2020). Nature Risk Rising: Why the Crisis Engulfing Nature Matters for Business and the Economy.

[5] https://www.worldbank.org/en/topic/climatechange/overview

[6] https://www.msn.com/en-gb/news/world/coronavirus-tracked-global-co2-emissions-fell-to-lowest-levelin-14-years-during-lockdowns/ar-BB14mRZY

[7] https://news.un.org/en/story/2018/09/1019472 